\title{
Evolution of Pulmonary Function Tests in Children with Complex Congenital Heart Disease and Scoliosis corrected by Posterior Approach.
}

Acevedo, $\mathrm{I}^{\star}$, Dominguez $\mathrm{F}^{\star}$, Burgos $\mathrm{J}^{\star \star}$, Escontrela $\mathrm{B}^{\star \star \star}$

*Ramon y Cajal Hospital, Dept of Anaesthesiology \& Intensive Care, Madrid, Spain, **Ramon y Cajal Hospital, Traumatology Dept, Madrid, Spain, ${ }^{* \star *}$ Cruces University Hospital, Dept of Anaesthesiology \& Intensive Care, Bilbao, Spain

The relationship between congenital heart disease and scoliosis is well studied, but the relationship between scoliosis surgery and evolution of pulmonary function tests is less known.

The aim of the study is to determine the evolution of respiratory function tests in patients with complex congenital heart disease and scoliosis corrected by posterior approach, for which the spirometric tests before surgery; one and two years after surgery were retrospectively reviewed. Data were analyzed with the Friedman test.

\begin{abstract}
Background and Goal:
The association between congenital heart disease and scoliosis is well studied. It is well known the relationship between spinal deformity and deterioration of lung function, which may stabilize after surgical correction.

The aim of this study is to determine the relationship between the degree of correction and the evolution of lung function tests at one and two years after corrective surgery in patients with congenital heart disease and scoliosis. We present this study as a continuation of a previously submitted
\end{abstract}

\begin{abstract}
Materials and Methods:
We retrospectively reviewed the records of 29 patients ( 19 female $/ 10$ male) with congenital heart disease which 9 were Tetralogy of Fallot, 8 univentricular hearts with Fontan, 3 with aortic coarctation, 4 ventricular septal defects, 1 truncus, 1 complete atrioventricular defect and 3 with aortic stenosis, all corrected before scoliosis surgery. All patients were surgically treated with segmental instrumentation by posterior procedures. Spinal cord monitoring and Transesophageal Echocardiography was used in all patients. Cobb, Pedriolle, spirometric values (preoperative (PRE), year and 2 years) and type of heart disease were compared with the Friedman test.
\end{abstract}

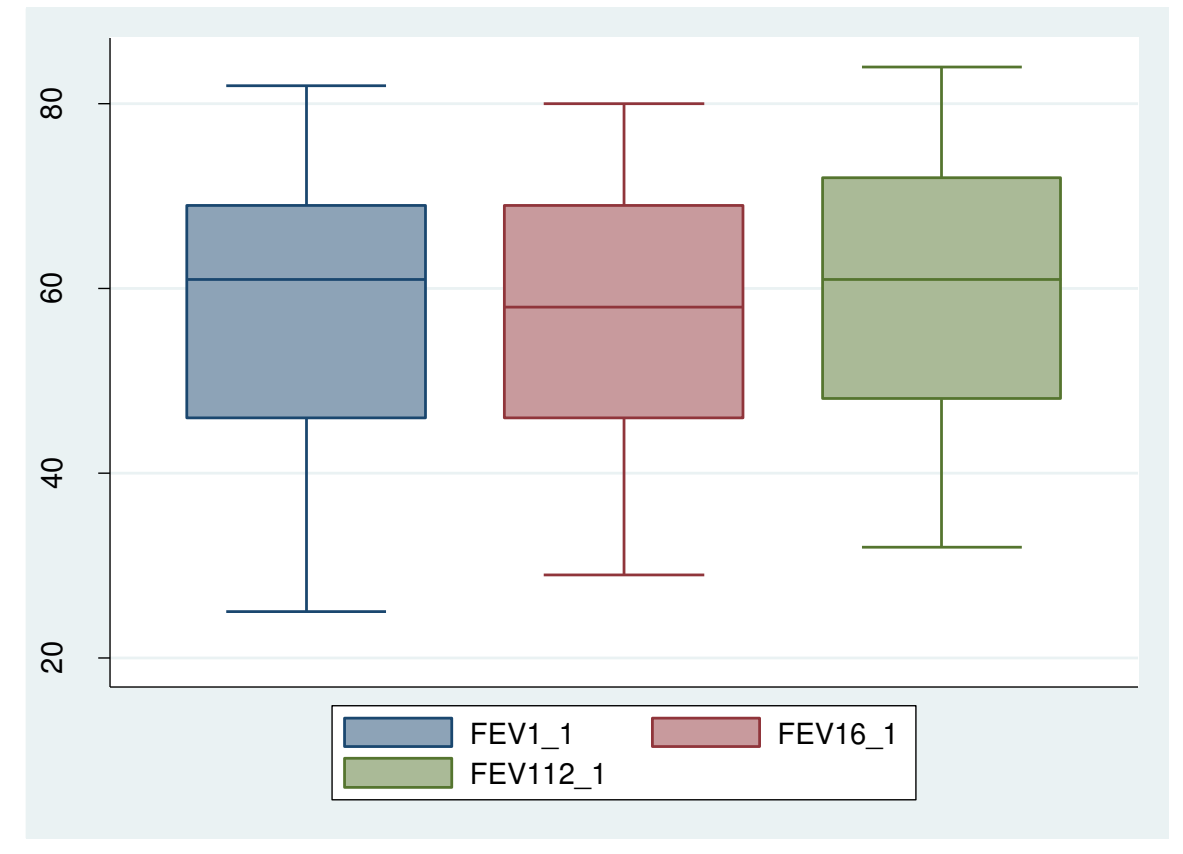

Results and Discussion:

Mean age was 17.3 years. Mean ejection fraction of the left ventricle (LVEF) was 57,6. American Society Anaesthesiologists score was III in 22 patients and IV in 7 and New York Heart Association score ranged was found between I and II. The PRE average Cobb was 65 and postoperative (PO) was 18. The average degree of PRE kyphosis T5T12 was 27.6 and 22.5 at 2 years. Percentage values of Forced Vital Capacity (FVC) and Forced expiratory volume in the first second (FEV1) showed improvement, being not significant for FVC (p 0,06) and significant for FEV1 (p 0,004). The improvement of FVE1/FVC was not significant. 10 patients had a PO spirometric pattern consistent with moderate restriction, 15 with severe restriction, 2 a mixed pattern and 2 a pattern consistent with normality. There was no relationship between type of heart disease and the spirometric improvement.

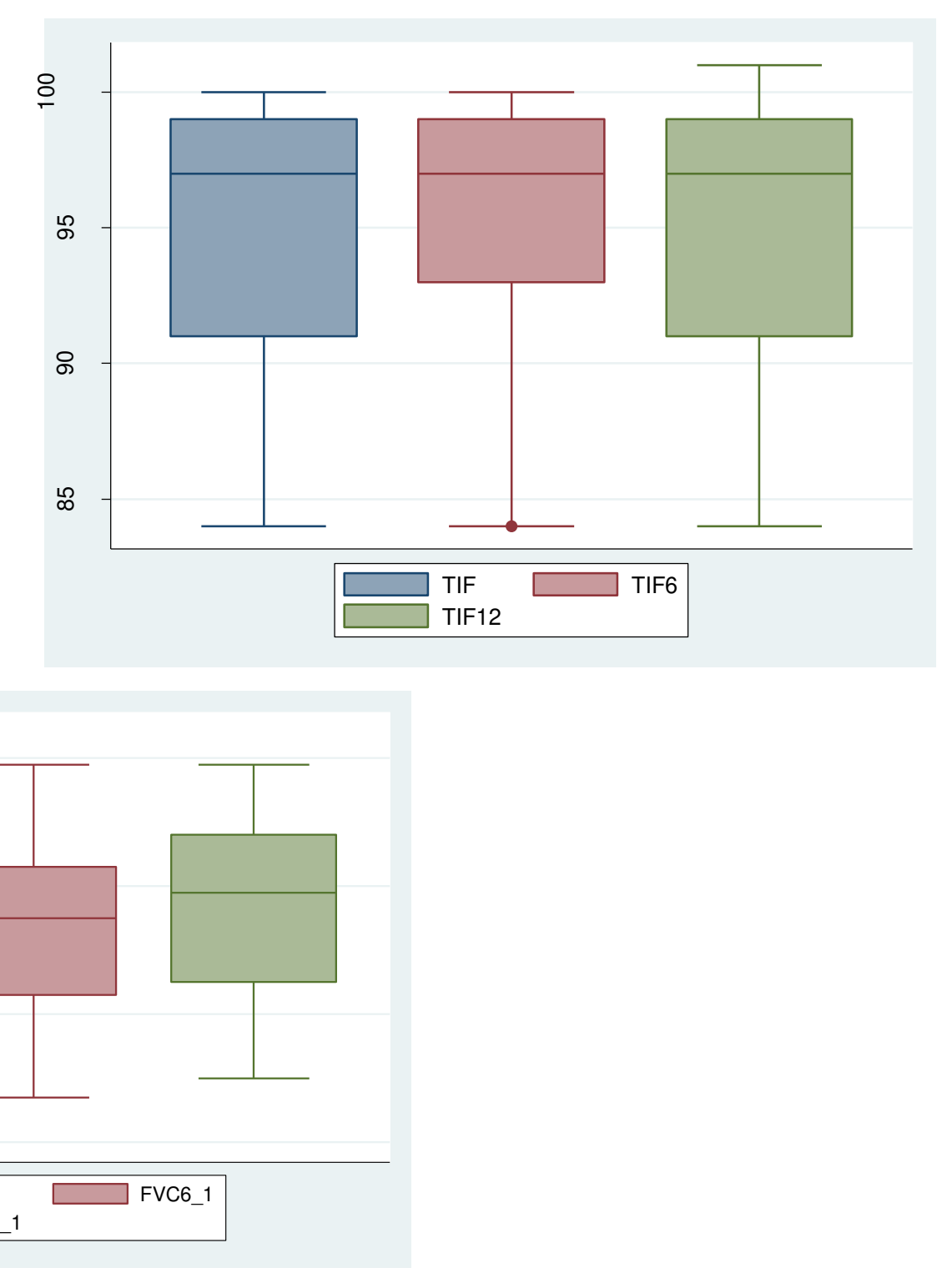

Conclusion:

There was no relationship between the type of heart disease and the evolution of spirometric tests. The spirometric impairment at 1 year could appear to be due to surgery, subsequently improved at 2 years

1. Jhonston, C. Richards, B. Sucato, C. Correlation of preoperative deformity magnitude and pulmonary function tests in adolescent idiopathic scoliosis. Spine 2011. 15;36(14):1096-102

2. Leichtle Cl1, Kumpf M, Gass M, Schmidt E, Niemeyer T. Surgical correction of scoliosis in children with congenital heart failure (Fontan circulation): case report and literature review. Eur Spine J. 2008 Sep;17 Suppl 2:S312-7 